\title{
The Association of TAS1R2 to Dental and Cardiovascular Health
}

\author{
Joseph Keryakos*, Annika van Oosbree, \& Alyssa Marie Kiesow \\ Department of Biology, Northern State University, Aberdeen, SD \\ https:/ / doi.org/10.33697/ ajur.2019.022 \\ Students:joseph.keryakos@wolves.northern.edu*,annika.vanoosbree@wolves.northern.edu \\ Mentor:alyssa.kiesow@northern.edu
}

\begin{abstract}
Despite brushing and/or flossing their teeth twice daily, many people are still susceptible to dental cavities and tooth decay. This research investigates the genetic and cardiovascular health behind this phenomenon. Two gene variants related to taste pathways, taste 1 receptor member 2 (TAS1R2) and taste 2 receptor member 38 (TAS2R38), were tested on the DNA of 20 students at Northern State University (10 males and 10 females). In concert with genetic screening, tooth impressions were taken of the participants' upper and lower jaws along with salivary $\mathrm{pH}$, heart rates, and blood pressures. Participants' cavities and fillings were counted and their gums examined for inflammation. Results showed that seven out of 10 males and two out of 10 females had the gene variant (TAS1R2). Students with this gene variant had an average salivary $\mathrm{pH}$ of 5.22 - significantly lower than the salivary $\mathrm{pH}$ for the other non-carrier students $(\mathrm{p}<0.05)$. These students also had smaller-sized tooth enamel, with none showing a size greater than one millimeter $(\bar{x}=0.84$ millimeters). Students not expressing the gene variant had fewer cavities than those expressing the TAS1R2 gene variant (i.e., one of the regions amplified). Four of the males and both of the females that carried the gene variant also showed signs of swollen gums, possibly contributing to heart disease in the future. Blood pressures and heart rates for the carriers were statistically significant $(\mathrm{p}<0.05)$, showing higher pressures and faster rates compared to non-carriers; meanwhile, all of the non-carriers had normal pressures and rates. Further, body mass index was lower among individuals without the gene variant. The results this limited study indicate that the TAS1R2 gene variant may play a role in cavity development and impact (or indicate poor) cardiovascular health, highlighting the importance of understanding the role of gene variants with regard to risk of tooth decay and gum and heart disease.
\end{abstract}

\section{KEYWORDS}

Dental cavities; Tooth decay; Gum and heart disease; Taste pathway gene; Gene variant; Blood pressure; Heart rate; Salivary pH; Tooth enamel

\section{INTRODUCTION}

Many people are still susceptible to dental cavities and tooth decay despite brushing and/or flossing their teeth twice daily. While dentists advise all people to brush their teeth and floss every day, this will not stop cavities from forming on their teeth; rather, it will just make it less likely to occur. Gene variants play a major role in the formation of dental caries. For example, a taste pathway gene variant can diminish oral and/or cardiovascular health. ${ }^{1}$ Furthermore, sweet taste preferences, also linked to taste pathway gene variants, can lower salivary $\mathrm{pH}$, which decreases the thickness of tooth enamel, making teeth more prone to cavities and decay. This subsequently leads to gum disease that could, later on, provoke heart disease.

Past studies examined the effects of four taste pathway gene variants associated with tooth decay and cavities. Wendell et al. (2010) researched the human taste receptor gene variants and showed that there were "statistically significant associations in taste pathway gene variants for caries risk." 1 They were able to find two genotypes that make the individual's diet rich in sugar and sweets - taste 2 receptor member 38, TAS2R 38 (7q34, 607751) and taste 1 receptor member 2, TAS1R2 (1p36.13, 606226). ${ }^{1}$ The genotypes transfer a signal to the brain notifying it of the sweetness of the food, and this leads the brain to favor sweet-flavored food. Hollá (2015) had a similar finding, determining that specific taste pathway gene variants lead an individual to prefer a diet rich in sugar. ${ }^{2}$ Each one of these gene variants causes the person to prefer sweet-tasting foods and can lead to higher chances of forming oral cavities.

Preferring sweet-tasting food is closely linked to lower salivary $\mathrm{pH}$. Food rich in sugar causes both an increase in oral bacteria that feed on the sweet food debris in the human mouth and a decrease in salivary $\mathrm{pH}^{3}$ Oral bacteria living in the human mouth favor the food debris that remains on the person's teeth and gums. Once the bacteria starts feeding on this debris, its product is acidic, thereby lowering salivary $\mathrm{pH}$ due to the process of sucrose fermentation. ${ }^{4}$ 
Acidic saliva caused by oral bacteria feeding on the debris of sweet food alters tooth enamel depth. Tooth enamel, the hard outer covering of the tooth, is mainly composed of minerals such as hydroxyapatite, an acid-soluble compound. ${ }^{5}$ Acidic saliva dissolves tooth enamel, ${ }^{6}$ and thinner enamel is associated with a higher risk of developing tooth caries. ${ }^{7}$ The acidic saliva in the mouth combines with the oral bacteria and food debris, forming a plaque that clings to the teeth. Further, thinner enamel with the presence of plaque causes caries that eventually lead to tooth decay. ${ }^{8}$

Dental caries are more prevalent in people carrying one of the aforementioned taste pathway gene variants because they are prone to the formation of plaque. Dental plaque remaining on teeth for more than three months hardens and cannot be removed by brushing, instead requiring scaling or planing. ${ }^{9}$ Rostami et al. (2017) concluded that, because of their propensity to develop/retain plaque, people expressing taste pathway gene variants should visit the dentist more than two times per year to prevent dental caries. ${ }^{9}$

Oral plaque causes three major damages: it reduces the size of the tooth enamel, induces the formation of dental caries, and instigates gingiva inflammation. ${ }^{10}$ Dental plaque contains bacteria that produce toxins, like butyrate and propionate, which can cause gum tissue to swell and bleed, or induce symptoms of gingivitis and periodontal disease. ${ }^{11}$ Additionally, there seems to be a link between the human immune system and the swollen gums. ${ }^{12}$ Once toxins from plaque contact the gums, the toxins can enter the bloodstream. Once the toxins are detected, the immune system sends phagocytes to ingest them, causing gums to swell and bleed.

Unfortunately, swollen gums are often a symptom of gum disease. Gum disease not only negatively affects oral health, but it may also disturb cardiovascular health. ${ }^{13}$ Once gum tissue is penetrated, bacteria can enter the bloodstream, circulate through the body into the heart, and accumulate in the carotid arteries, elevating blood pressure and heart rate and leading to heart diseases and stroke later on. ${ }^{14}$

The aforementioned research examines many aspects of dental health (i.e., taste gene variants in relation to tooth decay/plaque build-up, plaque build-up and gum health/immune system response, and oral health in relation to cardiovascular health); however, while all researched topics reported correlations, none sought to link all components together. Our research reported herein builds on existing data while gaining additional insight on correlations and relationships among taste pathway gene variants and physiological conditions in certain age groups. More specifically, our study examines the acidity of the saliva on teeth, screening for variants in two taste pathway gene variants, TAS1 and TAS2, to identify specific physiological limitations caused by these variants in male and female individuals ranging in age 18-23. Further, we seek to examine the link (if any) between tooth enamel depth and cavities as well as tooth decay, gum disease, and heart disease.

\section{METHODS AND PROCEDURES}

\section{Subjects}

Ten females and ten males (18 to 23 years old) of seemingly good health based on hair shininess, skin appearance, and weight, were asked to participate in this study. The Institutional Review Board of Northern State University approved our research on October 10, 2016 (IRB Protocol \#: 2016-09-28A) to examine a variety of physical attributes on human subjects. Participants voluntarily signed and submitted an informed consent stating the description of the research project, its risks and benefits, the time involvement, participants' rights, and the contact information of the research supervisors.

\section{Determining Gene Variants of Taste Pathway Genes}

Genomic DNA was isolated from at least $1 \mathrm{~mL}$ of saliva collected from each participant following protocol from previous research, ${ }^{15}$ albeit using $\mathrm{AB}$ lyses buffer (1 $\mathrm{M}$ Tris [pH 8.0], $5 \mathrm{M} \mathrm{NaCl}, 0.5 \mathrm{M}$ EDTA [pH 8.0], 10\% SDS) as a substitute for the procedural lyses buffer. Confirmation of DNA was conducted on a 1.0\% agarose $1 \mathrm{X}$ TBE gel using $\mathrm{EtBr}\left(\mathrm{Carolina}{ }^{\circledR}\right)$ as a nucleic acid stain in each sample and a water blank to test for contamination of products. Taste 2 receptor member 38, TAS2R 38 (7q34, 607751), and taste 1 receptor member 2, TAS1R2 (1p36.13, 606226), two known taste pathway gene variants, ${ }^{1}$ were selected for further analyses to identify amplifiable variants of these genes. Using Primer3 from IDT SciTools (bttp:// mmn.idtdna.com/SciTools/SciTools.asp x) we designed four primer pairs targeting gene variant regions of TASR38 and TAS1R2 taste pathway genes, then estimated annealing temperatures and levels of self-priming. IDT SciTools uses a suite of tools to adequately characterize sequences and customize primers. Four primer sets were developed and compared to target sequences using BLAST (reference) for specificity to detect a specific gene variant of two separate genes associated with taste perception (Table 1).

DNA Amplification

PCR was performed with $15 \mu \mathrm{L}$ reactions containing $50 \mathrm{ng}$ genomic DNA, $10 \mu \mathrm{M}$ of each primer (forward and reverse, Table 1), $400 \mu \mathrm{M}$ of each dNTP (dATP, dGTP, dCTP, and dTTP), 0.50 units of Taq polymerase in buffer (Promega), and $3 \mathrm{mM}$ of $\mathrm{MgCl}_{2}$. 
PCR started with initial denaturing at $94{ }^{\circ} \mathrm{C}$ for 4 minutes followed by 30 cycles of $94{ }^{\circ} \mathrm{C}$ for 1 minute, annealing at $53{ }^{\circ} \mathrm{C}$ for 1 minute, and extension at $72{ }^{\circ} \mathrm{C}$ for 1 minute. Confirmation of PCR amplification was conducted by running a sample on a $1.5 \%$ agarose $1 \mathrm{X}$ TBE gel using $\mathrm{EtBr}$ (Carolina ${ }^{\circledR}$ ) as a nucleic acid stain in each sample and a water blank to test for contamination of products in every PCR run. Even though real-time quantitative PCR could have quantified the gene variant expression, whether homozygous or heterozygous, presence of the gene variants was determined and compared to a negative control then quantified with an Invitrogen ${ }^{\circledR}$ Low DNA Mass ladder.

\section{Health Screening}

Several physiological attributes were tested to determine dental and cardiovascular health of participants in concert with genetic data. Salivary $\mathrm{pH}$ was measured with $\mathrm{pH}$ test strips. With an ultraviolet light, we examined the mouth of each participant for cavities, fillings and swollen gums. Finally, in order to measure the teeth enamel depth, we made tooth impressions of the upper and lower jaws using alginate and dental trays. Plaster was used to make the cast of the dental impressions and a Vernier ${ }^{\circledR}$ Caliper ruler was used to measure the width of the central incisors of each participant. Blood pressure and heart rates were measured twice a day (once in the morning before eating and once in the afternoon). Two readings of both heart rates and blood pressures were taken from each participant in order to get accurate results. Height and weight were also collected to determine the body mass index (BMI) of each participant.

Data Analyses

Data were analyzed using JMP® software (SAS Institute Inc.) to conduct comparisons via analysis of variance (ANOVA), where P-values less than 0.05 were considered significant.

\section{RESULTS}

Gene Variants of Taste Pathway Genes

Four primer sets were created for the purposes of this research (Table 1). The differences between these TAS gene variants were the product length and the variety of exons. For example, TAS2R38 had few but large exons while TAS1R2 had many but small exons. The TAS1R2.F1 and TAS1R2.R1 set had successful amplification, amplifying a gene variant of taste receptor 1 member 2 (TAS1R2, 1p36.13, 606226). This particular gene variant was found in seven out of 10 male participants and two out of 10 female participants. This gene variant was measured/quantified using low DNA mass ladder, and used hereafter for identifying physiology traits in relation to dental traits.

\begin{tabular}{|l|lll|}
\hline LOCI & PRIMER SEQUENCES (5'-3') & REGION & PRODUCT LENGTH \\
\hline TAS2R38.F1 & F: AGG CCC ACA TTA AAG CCC TC & Start: 803 Stop: & 204 bp \\
& & 822 & \\
TAS2R38.R1 & R: CAG CTC TCC TCA ACT TGG CA & Start: 1006 & \\
& & Stop: 987 & \\
\hline TAS2R38.F2 & F: GCA TCC GCA CTG TGT CCT AT & Start: 101 & 355 bp \\
& & Stop: 120 & \\
TAS2R38.R2 & R: AAA CGG ATG AGC TTG GAG CA & Start: 455 & \\
& & Stop: 436 & \\
\hline TAS1R2.F1 & GGC TGT GAC AAA AGC ACC TG & Start: 1211 & $\mathbf{7 5 0}$ bp \\
& & Stop: 1230 & \\
TAS1R2.R1 & GAA AGA ACG CAC GGC GAT AC & Start: 1960 & \\
& & Stop: 1941 & \\
\hline TAS1R2.F2 & CAG ATC ACC TAC AGC GCC AT & Start: 482 & 824 bp \\
& & Stop: 501 & \\
TAS1R2.R2 & TGG TCC AGG AGA GTG AA & Start: 1305 & \\
& & Stop: 1286 & \\
\hline
\end{tabular}

Table 1. A specification table of the four primer sets ( $\mathrm{F}=$ Forward, $\mathrm{R}=$ Reverse) designed during this research to isolate and amplify gene variants related to dental caries affiliated with taste receptors 1 and 2. We showed successful amplification of taste receptor 1 member 2 with primers TAS1R2.F1 and TAS1R2.R1 (in bold).

\section{Health Screening}

The average salivary $\mathrm{pH}$ of the participants with the gene variant TAS1R2 was 5.22 (SD \pm 0.45$)$, which is significantly lower than the average of the participants without the gene variant $(\bar{x}=6.72[\mathrm{SD} \pm 0.46] ; \mathrm{t}=7.4, \mathrm{df}=18, p<0.05)$. Median values are similar, where salivary $\mathrm{pH}$ is slightly acidic in those with the gene variant and salivary $\mathrm{pH}$ is neutral in those without the gene variant (Figure 1). Six out of the seven males with the gene variant TAS1R2 and both of the females with the gene variant had thin enamel $(0.84 \mathrm{~mm}$ [SD \pm 0.09$])$, while all of the individuals without the gene variant had an average thickness of $1.17 \mathrm{~mm}$ (SD \pm 0.1 , which is considered normal and healthy. Enamel thickness is significantly different between those with and without the gene variant $(\mathrm{t}=7.3 \mathrm{df}=17, p<0.05 ;$ Figure $\mathbf{1})$ 


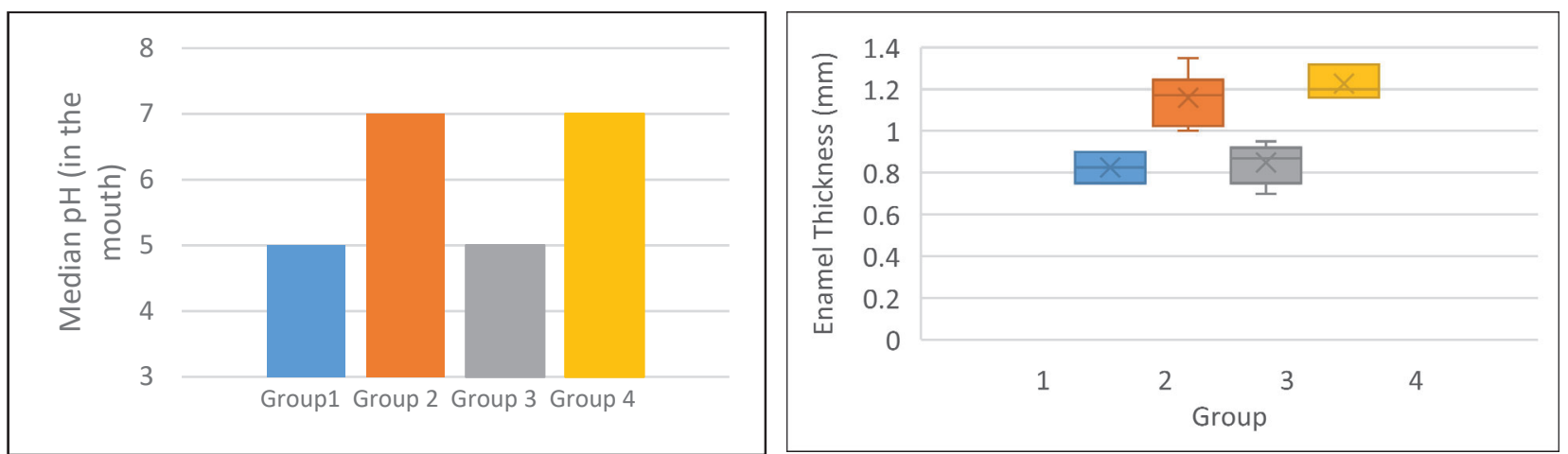

Figure 1. Left: Median salivary $\mathrm{pH}$ for female with the gene variant TAS1R2 (TAS) or Group 1, female individual without the gene variant TAS1R2 (Non-TAS) or Group 2, male with the gene variant TAS1R2 (TAS) or Group 3, male individual without the gene variant TAS1R2 (Non-TAS) or Group 4. Right: Enamel thickness (in $\mathrm{mm}$ ) for female with the gene variant TAS1R2 (TAS) or Group 1, female individual without the gene variant TAS1R2 (Non-TAS) or Group 2, male with the gene variant TAS1R2 (TAS) or Group 3, male individual without the gene variant TAS1R2 (Non-TAS) or Group 4. On the box and whisker plots, the line indicates median and $\mathrm{X}$ indicates mean. For both data figures, there is a difference between these attributes among these groups ( $\mathrm{p}<0.05)$.

The seven male participants with the gene variant TAS1R2 had an average of 11.86 cavities $(\mathrm{SD} \pm 7.14)$ with a median of 12 , while the three males without the gene variant TAS1R2 had a median of four cavities. The two female participants with the gene variant had an average and median of 6.5 cavities; one female had seven cavities while the other had six. The eight females without the gene variant TAS1R2 had a median of three cavitites. There is a significant difference in prevalence of cavities between individuals with the gene variant and those without the gene variant $(\mathrm{t}=-4.6, \mathrm{df}=10, p<0.05$; Figure 2$)$.

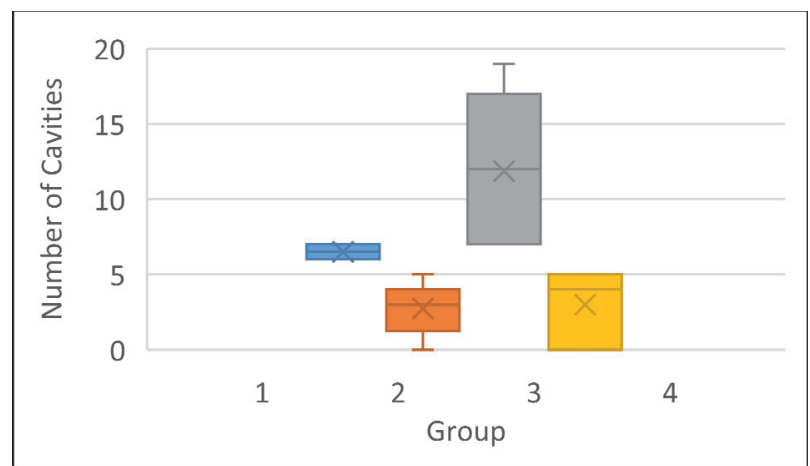

Figure 2. Number of cavities for female with the gene variant TAS1R2 (TAS) or Group 1, female individual without the gene variant TAS1R2 (Non-TAS) or Group 2, male with the gene variant TAS1R2 (TAS) or Group 3, male individual without the gene variant TAS1R2 (Non-TAS) or Group 4. There is a difference between these attributes among these groups $(\mathrm{P}<0.05)$. On the box and whisker plots, the line indicates median and $\mathrm{X}$ indicates mean.

Four out of the seven male participants with the gene variant TAS1R2 showed some gum swelling and were symptomatic for gum disease. Both female participants with the gene variant had swollen gums. None of the participants without the gene variant had a sign of gum disease or inflammation.

The seven males with the gene variant TAS1R2 had slightly high blood pressure levels $(\overline{\mathrm{x}}=127.14 / 82.86$ [SD \pm 6.9$])$ and heart rates $(\bar{x}=87[S D \pm 6.1], \tilde{x}=77)$. Both females with the gene variant had slightly high blood pressure levels $(\bar{x}=125 / 90[S D \pm$ 6.5]) and heart rates $(\bar{x}=86$ [SD \pm 6.01$], \tilde{x}=86)$. In all instances, participants without the gene variant showed normal blood pressure levels $(\bar{x}=115.45 / 72.72[S D \pm 6.86])$ and heart rates $(\bar{x}=62.90[\mathrm{SD} \pm 6.11], \tilde{x}=62$ [females], $\tilde{x}=60$ [males]). Heart rates $(\mathrm{t}=-6.1 \mathrm{df}=13, p<0.05$, Figure 3$)$ and blood pressure levels $(\mathrm{t}=-3.5 \mathrm{df}=14, p<0.05)$ were significantly different between those with and without the gene variant. 


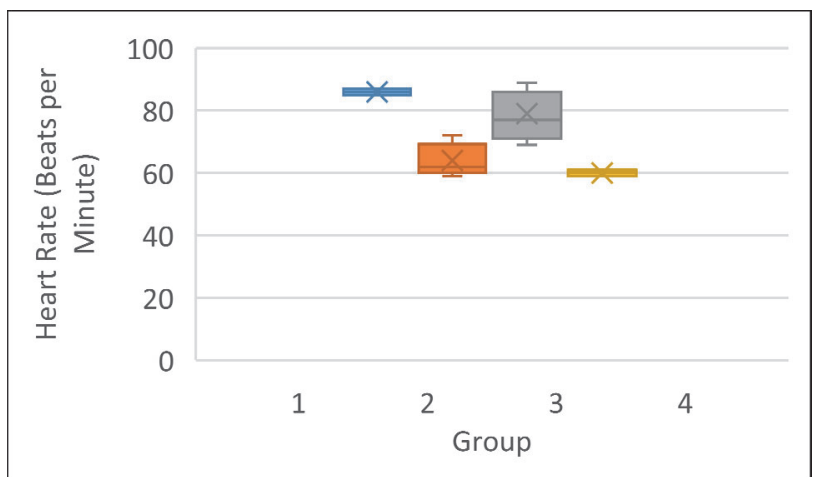

Figure 3. Heart rates for female with the gene variant TAS1R2 (TAS) or Group 1, female individual without the gene variant TAS1R2 (Non-TAS) or Group 2, male with the gene variant TAS1R2 (TAS) or Group 3, male individual without the gene variant TAS1R2 (Non-TAS) or Group 4. There is a difference between these attributes among these groups $(\mathrm{p}<0.05)$. On the box and whisker plots, the line indicates median and $\mathrm{X}$ indicates mean.

BMI values ranged from 16.5 to 24 in males and females. Males and females with the gene variant TAS1R2 had a slightly higher BMI (females, $\bar{x}=24$ [SD \pm 1 ],$\tilde{x}=24$; males, $\bar{x}=24.88$ [SD \pm 1.9$], \tilde{x}=25.2$ ) than those without the gene variant. Male and female participants without the gene variant showed normal BMI (females, $\bar{x}=17$ [SD \pm 0.6$], \tilde{x}=17$; males, $\bar{x}=18.77$ [SD \pm 1.6], $\tilde{x}=18.3)$. BMI was significantly different between those with and without the gene variant, where individuals without the variant showed lower BMI (Figure 4).

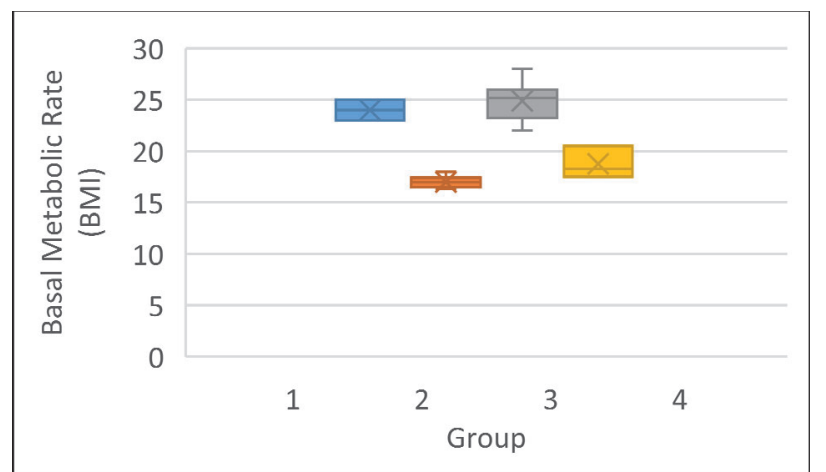

Figure 4. Body mass index (BMI) for female with the gene variant TAS1R2 (TAS) or Group 1, female individual without the gene variant TAS1R2 (Non-TAS) or Group 2, male with the gene variant TAS1R2 (TAS) or Group 3, male individual without the gene variant TAS1R2 (Non-TAS) or Group 4. There is a difference between these attributes among these groups $(\mathrm{p}<0.05)$. On the box and whisker plots, the line indicates median and $\mathrm{X}$ indicates mean.

\section{DISCUSSION}

Here, we provide evidence for an association between a specific gene variant, TAS1R2, and state of dental and cardiovascular health. Our data suggest that the presence of certain alleles for taste pathway genes, most notably the TAS1R2 gene variant, has an effect on dental and cardiovascular health. All participants at Northern State University presenting the TAS1R2 gene variant had poor dental health (i.e., low salivary $\mathrm{pH}$ and high numbers of dental caries) and subpar cardiovascular health (i.e., high blood pressure and heart rate) compared to individuals without the presentation of TAS1R2. Further, BMI was slightly higher in individuals with the TAS1R2 gene variant.

Specific single nucleotide polymorphisms (SNPs) of TAS1R2 have been linked with a susceptibility of developing dental caries. ${ }^{2}$ Specifically, the Val allele of rs35874116 (Ile191Val) was linked to overall dental development of dental caries. A study conducted on a specific population in West Mexico found that the Val191Val polymorphism in TAS1R2 is linked to high carbohydrate intake and dyslipidemia. ${ }^{16}$ Ramos-Lopez et al. (2016) hypothesized that "the Ile191Val polymorphism resides in the predicted first large extracellular domain of the TAS1R2 receptor, which hypothetically contains the ligand-binding site for carbohydrates and dipeptide sweeteners."'16 Additionally, compared with both Ile/Ile and Ile/Val polymorphisms, Val/Val convened a significantly higher prevalence of hypertriglyceridemia — a disorder, which is associated with atherosclerosis and may predispose a patient to developing heart disease..$^{16}$ This finding, paired with those stating this same polymorphism is linked to high carbohydrate intake, correlates with the findings of this study. Carriers of the TAS1R2 gene variant have lower salivary $\mathrm{pH}$, thinner enamel, more cavities, and higher blood pressure and heart rate (all with statistical significance). 
The presence of the TAS1R2 gene variant seems to initiate a cascade of events that can lead to tooth decay. This genotype facilitates the transfer of a signal to the brain indicating that the consumed food is very sweet. ${ }^{1}$ Based on the results of the present study involving salivary $\mathrm{pH}$, it is likely that the signal increases the brain's affinity for that particular sweetness of food, making future consumption of sweet food more likely. To our knowledge, this is the first study to study and identify a statistically significant link between the TAS1R2 variant and salivary $\mathrm{pH}$, potentially highlighting how this gene variant initiates this cascade that leads to tooth decay. Salivary $\mathrm{pH}$ lowers when sweet food is consumed due to bacteria feeding on the debris of the sweet food; ${ }^{3}$ therefore, a significantly lower salivary $\mathrm{pH}$ in the present study indicates higher consumption of sweet food. Acidic saliva eats away at the enamel, ${ }^{6}$ and, not surprisingly, the carriers of the TAS1R2 gene variant had significantly thinner enamels. Moreover, as chronic consumption of sweet food occurs, cavity development becomes more likely, as evidenced by the significantly higher number of cavities in those with the gene variant of TAS1R2 versus those without. This is in part due to the thinner enamel from the lower salivary $\mathrm{pH}$, as thinner enamel has been connected with a higher risk of developing caries. ${ }^{7}$ Ultimately, this could lead to gum disease or periodontitis, as both females and four out of the seven males with the gene variant show signs of one or both of these oral issues. Overall, a simple inherent affinity for sweet-flavored foods due to the TAS1R2 gene variant can lead to a cascade of events eventually ending in gum disease; the lower salivary pH subsequently leads to thinner enamel and more cavities.

This cascade can continue on to affect cardiovascular health. A strong relationship has been previously shown to exist between periodontitis and atherosclerotic vascular disease (ASVD), with patients with cardiovascular diseases having a higher percentage of caries, tooth loss, and orofacial pain. ${ }^{17}$ Heart rates and blood pressures collected during the present study demonstrated a close relationship between caries and elevated blood pressures and heart rates, with students presenting symptoms of gum disease having higher heart rates and blood pressures than participants with healthy gums $(p<0.05)$. This finding is likely due to the heavier prevalence of gum inflammation and evidence of future gum disease found at significantly higher levels in individuals with the gene variant. Once the gum tissue is penetrated, bacteria can easily enter the bloodstream and eventually lead to the accumulation of plaque in the lumen of the carotid arteries. ${ }^{14}$ This then causes the elevation in the patient's heart rate and blood pressure level—an elevation that could lead to ASVD and provoke a stroke later on. Eventually, the presence of the TAS1R2 gene variant creates an oral environment with a lower salivary $\mathrm{pH}$ due to an increased consumption of sweet food, after which a cascade of physiological events (due to lower salivary $\mathrm{pH}$ ) can lead to cardiovascular issues. The significantly higher heart rates and blood pressure levels found in those with versus without the gene variant confirm this relationship.

BMI plays a role in cardiovascular health. Research has shown that as BMI increases the risk of cardiovascular disease increases in both adults and children. ${ }^{18}$ Our research links dental health and the presence of the gene variant to cardiovascular health. Thus, the presence of the TAS1R2 gene variant may increase the chances of poor dental health and cardiovascular disease.

Regions attempted for amplification of TAS2R 38 contain a large continuous exon, while TAS1R2 is comprised of many smaller exons. Successful amplification of TAS1R2 captured multiple exons with only one of the two primer sets. It is likely that the second set of primers did not amplify the TAS1R2 because the primers targeted a larger exon region with minimal variation much like the primer sets for TAS1R38. We suggest targeting regions with more variation in exon length. Also, we amplified each DNA sample at least twice to ensure accuracy of identifying mutant versus wild-type (or presence versus absence) of the TAS1R2 gene variant.

Despite our study's statistically significant results, it had some limitations. The small sample size of 20 students was the major limitation of this study. In addition, the age demographic of college student participants was skewed to represent a younger population. Also, our study had some technical challenges as a limitation that could have interfered with the validity of our results. College students tend to have poorer oral health due to their diet and their failure to maintain good oral hygiene habits. In addition, tobacco use and caffeine intake were not assessed in this study. Another technical challenge could have been the blood pressures that were taken. The high blood pressures could have resulted from different factors not related to the gene variants such as caffeine consumption, physical exercise, medications, or from the White Coat Syndrome. Finally, teeth impressions, caries counting, and enamel measuring were not performed by a professional dental specialist. Therefore, the number of cavities and the enamel sizes could have been somewhat skewed. In future studies, a dentist could be employed to perform a professional dental exam.

\section{CONCLUSION}

To our knowledge, no studies have been conducted to date on whether a connection exists between TAS receptors and cardiovascular health. The present study offers preliminary data, albeit on a small sample, on the potential link between the TAS1R2 variant and signs of future heart disease. Further, our findings seem to support previous findings where females have a lower prevalence of periodontitis than males, ${ }^{19}$ which may suggest a link between gene variant expression, sex, and cardiovascular 
health. Perhaps future research could target the potential mechanisms behind the difference in rates of periodontitis between the sexes further to determine if this is primarily due to genetic reasons, e.g., TAS1R2 variant, or some other external factors.

In the future, this research could lead to further experiments on the taste pathway gene variants that are specifically associated with dental caries, gum disease, and heart disease. Variants of TAS1R2 could be genotyped and used to characterize large datasets according to expected and observed values as it relates to Hardy-Weinberg probabilities. As mentioned earlier, this research highlights the relevance of future studies on TAS1R2 and cardiovascular disease. In addition, genetic screenings for TAS1R2 gene variants could be conducted to assist with preventative dental measures and give indication of pre-existing health conditions (cavities, gum disease, and heart disease). Rostami et al. (2017) stated that people presenting the gene variant have to visit the dental office more often than non-expressers in order to eliminate the plaque on their teeth that leads to the formation of cavities. ${ }^{9}$ Screening kits could be assembled and sold to dental clinics, or a screening service could be provided to dental clinics, dentists, dental hygienists, and dental labs. These screenings could help practitioners and insurance companies identify patients that are more prone to cavities, gum disease, and heart disease, and help create more preventative, proactive dental plans for these individuals.

\section{ACKNOWLEDGEMENTS}

Authors thank Northern State University for the resources and assistance in conducting this research project and the Northern State University Institutional Review Board for approving this project. Additionally, authors thank the twenty participants for volunteering their time, saliva, and any inconvenience experienced while getting their teeth impressions taken for the benefit of this research.

\section{REFERENCES}

1. Wendell, S., Wang, X., Brown, M., Cooper, M. E., DeSensi, R. S., Weyant, R. J., Crout, R., McNeil, D. W., and Marazita, M. L. (2010) Taste genes associated with dental caries, J Dent Res 89, 1198-1202. https://doi.org/10.1177/0022034510381502

2. Hollá, I. L., Borilova, L. P., Lucanova, S., Kastovsky, J., Musilova, K., Bartosova, M., Kukletova, M., Kukla, L., and Dusek, L. (2015) GLUT2 and TAS1R2 polymorphisms and susceptibility to dental caries, Caries Res 49, 417-424. https:// doi.org/10.1159/000430958

3. Gupta, P., Gupta, N., Pawar, P. A., Birajdar, S. S., Natt, S. A., and Singh, P. H. (2013) Role of sugar and sugar substitutes in dental caries: a review, International Scholarly Research Notices: Dentistry 2013, 1-5. https:// doi.org/10.1155/2013/519421

4. Selwitz, H. R., Ismail, I. A., and Pitts, B. N. (2007) Dental caries, The Lancet 369, 51-59. https://doi.org/10.1016/S01406736(07)60031-2

5. Bartlett, D. W., Lussi, A., West, N. X., Bouchard, P., Sanz, M., and Bourgeois, D. (2013) Prevalence of tooth wear on buccal and lingual surfaces and possible risk factors in young European adults, J Dent 41, 1007-1013. https://doi.org/10.1016/j.jdent.2013.08.018

6. Zawaideh, F. I., Owais, A. I., and Mushtaha, S. (2017) Effect of CPP-ACP or a potassium nitrate sodium fluoride dentifrice on enamel erosion prevention, J Clin Pediatr Dent 41, 135-140. https:// doi.org/ 10.17796/1053-4628-41.2.135

7. Curson, M. E. J., and Duggal, M. S. (2003) Dental Disease - Structure of Teeth, in Encyclopedia of Food Sciences and Nutrition (Caballero, B., Ed.) 2nd ed., 1743-1746, Academic Press, New York. https:/ / doi.org/10.1016/B0-12-227055-X/00324-2

8. Marsh, P. D. (2010) Microbiology of dental plaque biofilms and their role in oral health and caries, Dent Clin North Am 82, 4 8. https:// doi.org/10.1016/j.cden.2010.03.002

9. Rostami, N., Shields, R. C., Yassin, S. A., Hawkins, A. R., Bowen, L., Luo, T. L., Rickard, A. H., Holliday, R., Preshaw, P. M., and Jakubovics, N. S. (2017) A critical role for extracellular DNA in dental plaque formation, J Dent Res 96, $208-216$. bttps:// doi.org/10.1177/0022034516675849

10. Hujoel, P. P., and Lingström, P. (2017) Nutrition, dental caries and periodontal disease: a narrative review, J Clin Periodontol 18, S79-S84. https://doi.org/10.1111/jcpe.12672

11. Singer, R. E., and Buckner, B. A. (1981) Butyrate and propionate: important components of toxic dental plaque extracts, Infect. Immun 32, 458-463.

12. Jepsen, S., Blanco, J., Buchalla, W., Carvalho, J. C., Dietrich, T., Dörfer, C., Eaton, K. A., Figuero, E., Frencken, J. E., Graziani, F., Higham, S. M., Kocher, T., Maltz, M., Ortiz-Vigon, A., Schmoeckel, J., Sculean, A., Tenuta, L. M., van der Veen, M. H., and Machiulskiene, V. (2017) Prevention and control of dental caries and periodontal diseases at individual and population level: consensus report of group 3 of joint EFP/ORCA workshop on the boundaries between caries and periodontal diseases, J Clin Periodontol 18, S85-S93. bttps:// doi.org/10.1111/jcpe.12687

13. Dhadse, P., Gattani, D., and Mishra, R. (2010) The link between periodontal disease and cardiovascular disease: How far we have come in last two decades? J Indian Soc Periodontol 14, 148-154. https:/ / dx.doi.org/10.4103\%2F0972-124X.75908

14. Humphrey, L. L., Fu R., Buckley, D. I., Freeman, M., and Helfand, M. (2008) Periodontal Disease and Coronary Heart Disease Incidence: A Systematic Review and Meta-analysis, J Gen Intern Med 23, 2079-2086. https://doi.org/10.1007/s11606008-0787-6 
15. Hearn, R. P., and Arblaster, K. E. (2010) DNA Extraction techniques for use in education, Biochemistry and Molecular Biology Education 38, 161-166. https://doi.org/10.1002/bmb.20351

16. Ramos-Lopez, O., Panduro, A., Martinez-Lopez, E., and Roman, S. (2016) Sweet taste receptor TAS1R2 polymorphism (Val191Val191) is associated with a higher carbohydrate intake and hypertriglyceridemia among the population of West Mexico, Nutrients 8, 101. bttps://doi.org/10.3390/ nu8020101

17. Dietrich, T., Webb I., Stenhouse, L., Pattni, A., Ready, D., Wanyonyi, K. L., White, S., and Gallagher, J. E. (2017) The relationship between oral and cardiovascular disease, Br Dent J 222, 381-385. https:/ / doi.org/ 10.1038/sj.bdj.2017.224

18. Friedemann, C., Heneghan, C., Mahtani, K., Thompson, M., Perera, R., and Ward, A. (2012) Cardiovascular disease risk in healthy children and its association with body mass index: systematic review and meta-analysis, BMJ 345, 1-16. bttps://doi.org/10.1136/bmj.e4759

19. Shiau, H. M., and Reynolds, M. A. (2010) Sex differences in destructive periodontal disease: a systematic review, J Periodontol 81, 1379-1389. https:/ / doi.org/ 10.1902/jop.2010.100044

\section{ABOUT THE STUDENT AUTHORS}

Joseph Keryakos graduated from Northern State University in Aberdeen, SD, on August 18, 2017, with a Bachelor of Science Degree in Biology In Honoribus and with a minor in Chemistry. Joseph is currently a third year Doctor of Pharmacy (Pharm.D.) candidate at South Dakota State University in Brookings, SD. He is also working as a clinical pharmacy intern at Avera McKennan Hospital \& University Health Center in Sioux Falls, SD. Annika van Oosbree graduated from Northern State University in May 2018 with a Bachelor of Science in Biology In Honoribus and in August 2018 with a Bachelor of Arts in English with a literature emphasis. She is currently a first year $\mathrm{MD} / \mathrm{PhD}$ candidate at the University of South Dakota Sanford School of Medicine in Vermillion, SD.

\section{PRESS SUMMARY}

Despite brushing and/or flossing their teeth twice daily, many people are still susceptible to dental caries and tooth decay. This research investigated the genetics behind this phenomenon. Two gene variants were tested, taste 1 receptor member 2 (TAS1R2) and taste 2 receptor member 38 (TAS2R38). One gene variant, TAS1R2, may show a link between its presence and dental and cardiovascular health. Though we only sampled 20 individuals (10 males and 10 females), our results indicate that the TAS1R2 gene variant may play a role in cavity development and cardiovascular health. 